\title{
International Symposium "Cultivation of marine organisms and its importance for marine biology": Opening address
}

\author{
O. KINNE \\ Biologische Anstalt Helgoland (Zentrale); Hamburg 50, Germany (FRG)
}

\section{Ladies and Gentlemen!}

My colleagues at the Biologische Anstalt Helgoland and I are grateful that you have accepted our invitation. All of us welcome you most cordially to this island and to our Marine Station. We are happy that some 258 scientists, representing 29 countries, have come here in order to present and to critically discuss new information on Cultivation of marine organisms and its importance for marine biology. It is both an honour and a pleasure for me to welcome you also on behalf of the Bundesregierung, the Government of the Federal Republic of Germany.

The considerable, world-wide interest in this International Helgoland Symposium has led to an unusually large number of applications for presentation of papers. Even though we have attempted to exclude all contributions not immediately relevant to the topic of this meeting, some 64 papers* will be presented, and additional accounts are scheduled to be offered during two Informal Sessions on Cultivation of plankton populations and on Fish-farming, including farming of other organisms of economic importance.

Such Informal Sessions represent a new element in the series of International Helgoland Symposia which was initiated in 1963. It is hoped that the Informal Sessions will provide an opportunity for discussing and evaluating important relevant problems more thoroughly and in greater depth than is possible during the restricted periods of time available after the presentation of a formal paper. We feel that a combination of formal papers and extensive informal discussions of specific issues offers maximum possibilities for exchange and assessment of information. Instead of recording and printing the discussions following the formal papers, we shall, this year, record the discussions held during the Informal Sessions and later print an

\footnotetext{
* Some of the papers presented at the symposium are not included in this volume.
} 
abbreviated version of these discussions which will be edited by the respective convener.

The problem of arranging the formal papers in some reasonable sequence turned out to be more difficult to solve than a rather odd crossword puzzle. Firstly, several speakers indicated they would prefer to present their paper at a specific time or on a specific day; secondly, all papers immediately related to either of the Informal Sessions had to be scheduled so as to precede that session in order to ensure maximum benefit; thirdly, there is only a total of $31 / 2$ days available! The only solution to the puzzle, that appeared to be acceptable, was to hold parallel sessions.

Parallel sessions provide advantages: (1) The rather large audience is divided into smaller groups; (2) it becomes possible to concentrate on specific aspects and to discuss them more effectively. Unfortunately, it was not possible to avoid parallel sessions concerned with quite similar topics. On the third day, for example, there will be parallel sessions on plants. Such overlapping is sincerely regretted but could not be avoided. We have, however, attempted to reduce such disadvantages of parallel sessions with closely related topics by means of subject differentiation and synchronous breaks which allow mutual auditor permeability. The need to hold parallel sessions has another consequence: there will be no translation service this year. The cost of employing simultaneously up to 3 such service is prohibitive. However, I am sure we will get along by making use of the new international scientific language, which is really quite similar to English.

This reference to the English language reminds me of a recent faculty meeting at the University of Kiel, North Germany. One professor seriously suggested that Latin be re-introduced as the international communication medium in science; immediately, a second professor exclaimed: "But the modern language of science is English!"; whereupon a third professor remarked drily, "Bad English, dear colleague, bad English!" There have been other international congresses without translation services. In one case, a Japanese scientist had his paper carefully translated into English; after he had been reading it for several minutes, an American among the audience whispered into his neighbour's ear: 'You know, Japanese isn't such a difficult language, after all."”

And now, Ladies and Gentlemen, please let me add a few words about the topic of this symposium.

About $2 / 3$ of the earth's surface is covered by oceans and coastal waters. The seas are considered to be the cradle of life. They still harbour a vast multitude of different forms of life. For the biologist, the seas represent an unparalleled source of information. It is especially in the largely undisturbed marine habitats that man can hope to learn more about the laws and rules which govern the dynamics of organismic co-existence, and to appreciate the complex functions and structures of ecosystems still submersed in their ancient saline environment. The future of mankind depends, to a considerable extent, on such knowledge and on a wise use of the organic and inorganic resources of oceans and coastal waters.

The marine biologist strives for maximum understanding of life in the marine environment. In order to approach this goal, he proceeds primarily along 4 major avenues: (1) he records data and collects samples from the sea's surface; (2) he 
carries out observations and experiments beneath the surface employing modern technologies such as underwater television, SCUBA diving, robots and underwater laboratories; (3) he cultivates marine organisms in restricted sea areas, ponds, aquaria, etc., and conducts experiments under controlled environmental conditions on quantitative and qualitative aspects of organismic performance; (4) he analyses physiological, morphological, behavioral, biochemical and genetical processes and mechanisms of responses, regulations and adaptations.

Of these major avenues, cultivation of and experimentation on marine organisms under controlled conditions deserve special attention. Less than $2 \%$ of all known marine organisms can presently be reared throughout their whole life cycle under controlled conditions. Our insufficient capacity for cultivating marine, especially oceanic, organisms has created a severe "bottle-neck" in the flow of information on life in the seas. Personally, I would go so far as to say that all marine organisms on earth can be cultivated, provided we have sufficient knowledge ... and money. Unless proved wrong, such an optimistic view seems useful for re-activating cultivation research which has suffered so many drawbacks, and yielded so many negative results, often caused by complete inadequacy of methods, equipment and financial support. There can be no doubt that increase of our present knowledge on maintenance and breeding of important representatives of the marine food web is of utmost importance if man is to achieve forecasting, controlling and managing capacities with regard to life processes in oceans and coastal waters.

Cultivation of marine organisms involves basic and applied research, ecological and economical aspects.

Ecologically, a greater capacity for cultivating marine organisms is likely to yield better insight into (1) functional and structural responses to variations in environmental factor intensities; (2) intra- and interspecific population dynamics; (3) distribution and abundance of organic matter in time and space; (4) evolution of the vast diversity of marine life; (5) requirements for continued existence of single marine species and of species assemblages (ecosystems). Of special ecological importance are studies on life cycles and rates and efficiencies of metabolism (food consumption and conversion, growth, reproduction, respiration) of "mass species" and on dynamic aspects of experimental ecosystems. It is a prerequisite for such studies, that the experimenter be able to cultivate his research objects under defined environmental conditions.

Economically, a greater capacity to cultivate marine organisms helps to provide food and raw materials for the growing human population, and to open up alternatives to the primitive exploitation techniques, based on gathering, collecting and hunting, which still dominate the so-called "production" of sea food and other organic marine materials. Successful cultivation of algae, molluscs, crustaceans, fishes and other organisms of economic (commercial) importance provides possibilities for genetic stock improvement in terms of growth rates, taste, appearance, environmental resistance, etc., and may result in controlled domestication of certain marine organisms. In some cases, cultivation through the whole life cycle (including reproduction) may not be necessary for economic purposes. Whenever early ontogenetic stages are 
available with certainty and in adequate quantity and quality, "fattening" to market size may be sufficient.

In order to provide the biological know-how required for strengthening commercial mariculture, we must learn more about competition and intraspecific variation in the marine environment, about diseases of marine organisms, about optimum conditions for growth, reproduction and metabolic efficiency, about breeding organisms for restocking oceanic or littoral areas, and about producing organisms directly for marketing (fattening, domestication). The differences in the aims of the two last-mentioned points have scarcely been fully appreciated. Successful restocking requires different "end-products" than fattening or domestication. The first case calls for mass production of young life-cycle stages with a maximum potential for competition in the sea and with maximum physiological adjustment to the particular abiotic environment encountered upon release. The second case calls for mass production of fast growing, pleasant-tasting organisms, resistant to negative effects of domestication (e.g. space limitation, disease, water eutrophication or pollution) and with maximum efficiency of converting cheap food sources into body-own substances.

Organisms occupying low positions in the marine food pyramid, such as filter feeders, transfer planktonic primary producers, bacteria and non-living organic material directly into body-own substances, and hence can be farmed with greater efficiency in terms of food conversion than organisms at the top of the pyramid, such as carnivorous crustaceans and fishes, which require secondary or tertiary food web links as food sources. Technically, sessile forms (e.g. multicellular plants, sponges, and lamellibranchs) frequently require lower capital investments for equipment, buildings and space than motile forms. However, motile species, to which belong all commercially used crustaceans and fishes and many other marine organisms of economical importance, can also be farmed on a large scale basis in suitable sea areas at reasonable cost. Depending on coastal morphology, climate, natural food sources, water exchange and the species to be cultured, such farms can be operated in various ways by erecting fences ("fence techniques") or by imprinting the farmed organism to remain in a given locality until harvest time, making use of its behavioral properties ("behavior techniques"). Fence techniques are required for migrating forms or for organisms which must be protected from predators. They limit the space available to the organism by separating from the open sea protected semi-enclosed areas employing dams, sluices, nets, etc., or by the use of cages, tents and other in-situ cultivation devices below the seas' surface. Behavior techniques may be chosen for nonmigrating organisms which can be trained to remain in a given sea area, e.g. by employing automatic feeding devices and directional stimuli such as light, sound, odor, or electricity. Positive behavioral stimuli are used to attract the organism to the center of the individual farm area, negative stimuli to deter it from its (otherwise unmarked) borders or from entering adjacent farm sections. Preliminary experiments suggest that behavior techniques will assume increasing importance in the near future. We hope to hear more about these and related problems within the next few days.

In the long run, man cannot just continue to harvest the seas. The present trend and the ever-growing international potential for ruthless exploitation of the marine environment must, sooner or later, lead to irreversible damages in certain marine 
biota. Parallel developments in terrestrial and limnic habitats provide ample proof for such a statement. The benefit of continued use of the biological resources of oceans and coastal waters is no divine present, but has to be paid for in terms of responsible measures of conservation and management. Such measures must be based on scientific knowledge - much more knowledge than is presently available.

Ladies and Gentlemen, Colleagues and Friends! It is my sincere hope that this International Meeting will become a milestone in critically assessing our present body of knowledge on marine and brackish water aquaculture in the widest sense of that term, and in stimulating further research which is so urgently needed in this important field of modern marine biology.

With this hope, I herewith open the International Helgoland Symposium 1969 on Cultivation of marine organisms and its importance for marine biology. 